\title{
Características Físicas, Microbiológicas y Sensoriales de Queso Blanco Fresco y Salchicha Premium Bajas en Contenido de Sodio
}

\author{
Lina M. Suarez*, Leidy J. Gómez y José E. Zapata \\ Universidad de Antioquia UdeA, Grupo de Nutrición y Tecnología de Alimentos, Facultad de Ciencias \\ Farmacéuticas y Alimentarias, Calle 67 No. 53 - 108, Medellín - Colombia \\ (e-mail: linam.suarez@udea.edu.co; edgar.zapata@udea.edu.co; ljohanna.gomez@udea.edu.co) \\ ${ }^{*}$ Autor a quien debe ser enviada la correspondencia
}

Recibido May. 17, 2017; Aceptado Jul. 6, 2017; Versión final Sep. 6, 2017, Publicado Dic. 2017

\begin{abstract}
Resumen
Se ha evaluado el efecto de la sustitución de $\mathrm{NaCl}$ sobre propiedades físicas, sensoriales y microbiológicas de salchicha premium y queso blanco fresco. Se elaboraron salchichas y quesos con sustitución del $63 \%$ de $\mathrm{NaCl}$ por mezclas de sales (muestras A,B,C y D) y una de control $(100 \% \mathrm{NaCl}$ ). A los productos se les analizó color, perfil de textura, perfil sensorial y calidad microbiológica. Los productos mostraron comportamientos diferentes según la mezcla usada. Se observó que el color no presenta diferencia para salchicha $\mathrm{A}$ y queso $\mathrm{B}$, en tanto el perfil de textura tuvo un comportamiento similar para todas las sustituciones, sin embargo, sensorialmente queso $A$ y salchicha $D$ tuvieron calificación más baja en la aceptación general, mientras, la calidad microbiológica de los dos productos no se vio alterada por la sustitución. Los resultados sugieren que el contenido en iones de las sales afecta el perfil de textura y de sabor de los productos, pero no afecta la calidad microbiológica ni el color. Además es posible la sustitución del $63 \%$ de $\mathrm{NaCl}$ en la elaboración de salchichas y queso fresco, sin afectar sus características físicas, microbiológicas y sensoriales.
\end{abstract}

Palabras claves: reducción de sodio; caracterización física; caracterización microbiológica; queso fresco; salchicha

\section{Physical, Microbiological and Sensory Characteristics of Fresh White Cheese and Premium Sausage Low in Sodium}

\begin{abstract}
The effect of the substitution of $\mathrm{NaCl}$ on the physical, sensory and microbiological properties of premium sausage and cottage white cheese has been evaluated. Sausages and cheeses were made with $63 \% \mathrm{NaCl}$ substitution by salt mixtures (samples $A, B, C$ and D) and a control sample $(100 \% \mathrm{NaCl})$. The products were analyzed for color, texture profile, sensory profile and microbiological quality. The products showed different behavior depending on the mixture used. It was observed that color does not present differences for sausage $A$ and cheese $B$, while the texture profile had a similar behavior for all the substitutions. However, cheese $A$ and sausage $D$ had sensorial lower rating in the general acceptance, and the microbiological quality of the two products was not altered by substitution. The results suggest that the ion content of the salts affect the texture and taste profile of the products but does not affect microbiological quality or color. It is also possible to replace $63 \% \mathrm{NaCl}$ in the production of sausages and cottage cheese, without affecting their physical, microbiological and sensorial characteristics.
\end{abstract}

Keywords: sodium reduction; physical characterization; microbiological characterization; cottage cheese; sausage 


\section{INTRODUCCIÓN}

El cloruro de sodio o sal es un ingrediente ampliamente usado en la industria de alimentos, la sal tiene la capacidad de mejorar los sabores, prevenir el crecimiento microbiano y la actividad enzimática en productos procesados (Carabante, 2013). Sin embargo, durante las últimas décadas, algunos estudios indican un alto nivel de consumo de sodio vinculado a enfermedades renales y a incrementos en la presión arterial que afecta el riesgo de mortalidad por enfermedad cardiovascular y representan un serio problema de salud pública (Zhou et al., 2016). Actualmente, la ingesta de cloruro de sodio en países desarrollados varía entre 8 a $13 \mathrm{~g}$ por día, que es muy superior a los $5 \mathrm{~g}$ recomendados por la Organización Mundial de la Salud (Desmond, 2006). El 75\% de la ingesta de sodio se deriva de alimentos procesados, siendo los productos lácteos y embutidos cárnicos los de mayor influencia, indicando la necesidad de reducir el sodio durante su elaboración (Felicio et al., 2013), esto implica una reducción o sustitución por otros tipos de sales que deben satisfacer la necesidad de los consumidores en cuanto a calidad sensorial y características físicas y microbiológicas (Ganesan et al., 2014). La sustitución del cloruro de sodio ( $\mathrm{NaCl}$ ) por otras sales o su reducción, tiene implicaciones diversas en el procesamiento industrial, viéndose reflejado en las propiedades fisicoquímicas, microbiológicas, funcionales y sensoriales del producto (Carabante, 2013).

Las sustituciones de $\mathrm{NaCl}$ por sales de potasio son las más implementadas en la reducción del sodio. Se ha estudiado sustituciones de aproximadamente el $50 \%$ de $\mathrm{NaCl}$ por sales de $\mathrm{K}$ en queso mozzarella, el cual presentó una aceptación sensorial similar al control (Rodrigues et al., 2014). A estos mismos niveles de sustitución, el queso cheddar mostró una calidad sensorial aceptable pero con presencia de sabor amargo (Carabante, 2013). Para este mismo queso, cuando se utilizó una sustitución total de $\mathrm{NaCl}$ por sales de K, Mg o $\mathrm{Ca}$, el sabor amargo fue predominante, pero mezclado en una proporción 1:1 de sales de $\mathrm{K}$, Mg o Ca con $\mathrm{NaCl}$ se observó una mejora en la calidad sensorial, siendo menos salados los quesos elaborados con las mezclas que contenían sales de Mg y Ca (Fitzgerald y Buckley, 1985).

Así mismo, para embutidos cárnicos los estudios sugieren que es posible reducir la concentración de $\mathrm{NaCl}$ en un $50 \%$ en salchichas mediante el uso de sales de $\mathrm{K}$, sin cambios desfavorables en las características sensoriales y microbiológicas (Pacheco et al., 2012). Mientras que otras sustituciones con sales de Ca han afectado el color, con valores superiores de $a^{*} \mathrm{y}^{*}$, valores menores para $\mathrm{L}^{*}$, dureza y gomosidad en relación al control (Gimeno et al., 2001). También, fueron usadas mezclas de sales de $\mathrm{K}$ y Ca para sustituir el $40 \%$ del contenido de $\mathrm{NaCl}$ exhibiendo características similares de color y textura en comparación al control (100\% NaCl) (Choi et al., 2014).

Por lo tanto, si se quiere lograr una reducción del 30\% de Na o más, el sabor salado se debe mantener con alguna sustancia que no contenga sodio. Para reemplazar el sabor salado en productos con sodio reducido, otras sales de $\mathrm{K}, \mathrm{Mg}$, y Ca fueron utilizadas como sustitutos, sin embargo, estos no proporcionan el mismo nivel de salinidad que los iones de $\mathrm{Na}$ y algunos aportan malos sabores, tales como amargo o metálico, que limitan su aceptación sensorial (Carabante, 2013). Por lo anterior, el objetivo general de este estudio fue evaluar el efecto de la sustitución del $\mathrm{NaCl}$ sobre las características de textura, color, calidad microbiológica y sensorial de salchicha premium y queso fresco blanco.

\section{METODOLOGÍA}

La metodología presenta la composición de las mezclas de sales que se usaron para sustituir el $63 \%$ de $\mathrm{NaCl}$ en la elaboración de las salchichas y los quesos, la preparación de los productos, los análisis físicos, microbiológicos y sensoriales realizados.

\section{Mezclas sustitutas de cloruro de sodio}

Se obtuvieron 4 mezclas con una sustitución del $63 \%$ del $\mathrm{NaCl}$, a partir de un diseño de experimentos en el que se evaluaron 66 mezclas de sales, con diferentes niveles de iones $\left(\mathrm{Na}^{+}, \mathrm{K}^{+}, \mathrm{Ca}^{2+}\right.$ y $\left.\mathrm{Mg}^{2+}\right)$, escogiendo las 4 que tuvieran mejor perfil sensorial en solución acuosa (resultados no mostrados). Dichas mezclas se usaron para la preparación de salchichas premium y queso blanco fresco semiduro- semigraso. En la Tabla 1 se muestra el contenido de iones $\mathrm{Na}^{+}, \mathrm{K}^{+}, \mathrm{Ca}^{2+}$ y $\mathrm{Mg}^{2+}$ para cada una de las mezclas sustitutas (Bellchem, Colombia). Las mezclas fueron evaluadas sensorialmente con un panel experto de 6 jueces, el cual determinó intensidad de sabor salado (SS), intensidad de sabor amargo (SA), intensidad de sabor metálico (SM), sabor residual salado (SRS), sabor residual amargo (SRA), sabor residual metálico (SRM), además de sabor objetable (SO) y calidad general, por medio del perfile sensorial por aproximación multidimensional. 
Tabla 1: Composición iónica de las mezclas de sales sustitutas

\begin{tabular}{ccccc}
\hline MEZCLA & $\mathrm{Na}^{+}(\%)$ & $\mathrm{K}^{+}(\%)$ & $\mathrm{Ca}^{2+}(\%)$ & $\mathrm{Mg}^{2+}(\%)$ \\
\hline $\mathrm{A}$ & 14,54 & 11,14 & 5,40 & 2,46 \\
$\mathrm{~B}$ & 14,45 & 19,69 & 4,09 & 0,00 \\
$\mathrm{C}$ & 14,54 & 15,51 & 2,53 & 2,26 \\
$\mathrm{D}$ & 14,34 & 21,11 & 0,57 & 1,29 \\
\hline
\end{tabular}

\section{Preparación de las salchichas}

Se elaboraron $2 \mathrm{Kg}$ de salchicha premium por duplicado para cada una de las cuatro mezclas sustitutas y $\mathrm{NaCl}$ como control, usando la formulación que se presenta en la Tabla 2. La carne y grasa fueron procesadas en un molino de discos en un tamaño de poro de $2 \mathrm{~mm}$ y $4 \mathrm{~mm}$, respectivamente. La carne fue mezclada con los demás ingredientes en el siguiente orden de adición: aditivos solubles en agua, condimentos, grasa, harina y el hielo. Posteriormente la mezcla fue embutida en funda sintética y dividida en salchichas de aproximadamente $20 \mathrm{~g}$. Finalmente las salchichas fueron sometidas a una cocción seca hasta alcanzar $50-55^{\circ} \mathrm{C}$ en una cámara de cocción (Mauting, República checa) y una cocción húmeda de $72^{\circ} \mathrm{C}$.

Tabla 2: Formulación de salchicha Premium.

\begin{tabular}{lc}
\hline \multicolumn{1}{c}{ COMPONENTE } & PORCENTAJE (\%) \\
\hline Carne bovina & 56 \\
Pasta de pollo & 10 \\
Grasa & 7,70 \\
Ascorbato de sodio & 0,30 \\
Fosfato de sodio & 0,08 \\
Condimento salchicha & 1,00 \\
Proteína de soya & 2,50 \\
Harina de papa & 3,40 \\
Humo líquido & 0,04 \\
Nitral sal curante & 0,17 \\
Hielo & 18,17 \\
NaCl o mezcla sustituta & 0,60 \\
\hline
\end{tabular}

\section{Preparación de queso}

Se elaboró $2 \mathrm{Kg}$ de queso fresco blanco semiduro- semigraso por duplicado para cada una de las mezclas de sales y NaCl como control. La formulación usada fue: $100 \%$ de leche, $0,033 \%$ cuajo (fuerza de cuajo de $1: 22000), 0,2 \%$ de $\mathrm{CaCl}_{2}$ y $1,5 \%$ de las mezclas o $\mathrm{NaCl}$. La leche fue pasteurizada ( $\mathrm{T}: 60 \stackrel{\circ}{ } \mathrm{C}$ y t: $30 \mathrm{~min}$ ), posteriormente se enfrió hasta alcanzar $48^{\circ} \mathrm{C}$, se adicionó el $\mathrm{CaCl}_{2}$, cuando alcanzó una temperatura de $32^{\circ} \mathrm{C}$ se adicionó el cuajo. Se mezcló con un aspa por 1 minuto, se dejó en reposo durante 40 min, tiempo en el cual se formó el cuajo. Se cortó el cuajo para liberar el suero lácteo, se dejó reposar por 5 min, luego se calentó hasta $40^{\circ} \mathrm{C}$, se agitó por $10 \mathrm{~min}$ y los gránulos de cuajo fueron separados del suero lácteo. Finalmente, se adicionó la mezcla sustituta o el $\mathrm{NaCl}$, se moldeó y prensó el queso con una prensa hidráulica.

\section{Análisis microbiológico}

Se realizó la determinación de la calidad microbiológica de los productos elaborados con las cuatro mezclas y el $\mathrm{NaCl}$ por medio de los análisis exigidos por la norma NTC 1325: productos cárnicos procesados no enlatados para las salchichas, en la cual se analizaron microorganismo mesófilos (M), recuento de coliformes totales (CT), coliformes fecales (CF) y E. coli (EC), recuento de S. aureus cuagulasa positiva (SA), recuento de esporas de Clostridium sulfito reductores (C), detección de Salmonella sp. (S) y detección de L. monocytogenes (LM) (ICONTEC, 2008). La norma NTC 5894: productos lácteos para los quesos en la cual se analizaron recuento de mohos (Mo) y levaduras (L), CT y EC, SA, S y LM (ICONTEC, 2011). Se realizó por duplicado.

\section{Evaluación sensorial}

Se realizó un perfil sensorial de las salchichas y los quesos elaborados con las cuatro mezclas de sales y el $\mathrm{NaCl}$, por medio de una evaluación tipo descriptiva-cuantitativa según NTC 3932 y 5328, realizada por un panel de expertos de 6 jueces de la fundación INTAL. Las características sensoriales medidas fueron: apariencia (A), olor característico (OC), olor objetable (OO), sabor característico (SC), sabor objetable (SO), sabor salado (SS), sabor amargo (SA), sabor metálico (SM), cohesividad (C), masticabilidad (Ma), calidad general y aceptación. Además, para el queso se midió sabor acido (Ac), sabor graso (SG), y dureza (D). Estos fueron calificados en una escala de 10 puntos, donde 0 es ausente y 10 es muy marcado. 


\section{Análisis de color}

Los parámetros de color de las salchichas y los quesos elaborados con las cuatro mezclas de sales y el $\mathrm{NaCl}$, fueron determinados por medio de un espectrocolorímetro portátil de esfera SP-60 (X-Rite, USA), y los resultados se expresaron en términos de luminosidad $\left(\mathrm{L}^{*}\right)$, color rojo $\left(\mathrm{a}^{*}\right)$ y color amarillo $\left(\mathrm{b}^{*}\right)$, según lo recomendado por la Comisión Internacional de l'Eclairage (1979). Se realizó por sextuplicado para cada producto.

\section{Medición instrumental de textura}

Para los quesos y las salchichas elaborados se realizó un análisis de perfil de textura (TPA) por medio de un texturómetro modelo EZ-SX (Shimadzu, Japón) con una celda de $25 \mathrm{~kg}$. Las muestras de quesos fueron cortadas en cubos de $20 \mathrm{~mm}$ y las salchichas en cilindros de $10 \mathrm{~mm}$ de radio y $20 \mathrm{~mm}$ de altura. Las muestras fueron comprimidas en un $30 \%$ de su altura original utilizando un plato $\mathrm{P} / 75$, con una velocidad pre-ensayo, ensayo, post-ensayo de $50 \mathrm{~mm} / \mathrm{min}$ y un tiempo de reposo entre las compresiones de $5 \mathrm{~s}$. Durante el análisis fueron determinados los parámetros dureza $(N)$, elasticidad $(N / m)$, cohesividad, gomosidad (N) y masticabilidad (N). Se realizó por sextuplicado para cada producto.

\section{Análisis estadístico}

Se utilizó la prueba de la mínima diferencia significativa (LSD) para determinar diferencias entre las medias, con un nivel de confianza del $95 \%$. Los análisis fueron realizados en el paquete estadístico Statgraphics Centurion XV.

\section{RESULTADOS Y DISCUSIÓN}

Los resultados se organizan en cinco subsecciones: i) mezclas sustitutas de cloruro de sodio; ii) efecto de la sustitución del $\mathrm{NaCl}$ sobre la calidad microbiológica de los productos; iii) efecto de la sustitución del $\mathrm{NaCl}$ sobre las características sensoriales de los productos; iv) efecto de la sustitución del $\mathrm{NaCl}$ sobre el color; y v) efecto de la sustitución del $\mathrm{NaCl}$ sobre la textura instrumental.

\section{Mezclas sustitutas de cloruro de sodio}

Para comparar los resultados sensoriales de las mezclas de sales sustitutas, se usó una sal comercial baja en sodio como referencia,VITAL $40 \%$ menos sodio (BRINSA S.A., Colombia) y $\mathrm{NaCl}$ grado alimenticio (BRINSA S.A., Colombia) como control. El perfil de sabor de las mezclas sustitutas y las sales de referencia se presenta en la Tabla 3 y Fig. 1. Se observa, que excepto por la mezcla A, todas tienen un perfil de sabor similar a la del control; con una calidad sensorial de 7,5 para la sal de referencias Vital, 8,7 para $\mathrm{NaCl}, \mathrm{y}$ valores entre 6 y 7,2 para las mezclas de sales sustitutas.

Tabla 3: Perfil sensorial de sabor de las mezclas de sales sustitutas, la sal Vital $40 \%$ menos sodio y $\mathrm{NaCl}$ grado alimenticio.

\begin{tabular}{ccccccc}
\hline DESCRIPTORES & Control & Vital & $A$ & $B$ & $C$ & $D$ \\
\hline$\% \mathrm{NaCl}$ & 100 & 60 & 37 & 37 & 37 & 37 \\
SS & 8,9 & 8,6 & 7,4 & 8,2 & 6,6 & 7,1 \\
SA & 0,6 & 1,3 & 3,3 & 1,8 & 1,6 & 0,8 \\
SM & 0,4 & 1,4 & 2,7 & 1,8 & 1,6 & 1,0 \\
SO & 0,1 & 0,3 & 0,6 & 0,4 & 0,3 & 0,0 \\
SRS & 6,6 & 4,9 & 2,1 & 4,8 & 2,8 & 2,9 \\
SRA & 0,4 & 1,0 & 2,0 & 0,9 & 0,4 & 0,3 \\
SRM & 0,7 & 1,0 & 2,3 & 1,2 & 0,7 & 0,7 \\
Calidad general & 8,7 & 7,5 & 6,6 & 6,8 & 6,0 & 7,2 \\
\hline
\end{tabular}

Entre los resultados se puede destacar que con la mezcla $D$ se logró obtener una sal sustituta que además de contener menos sodio, presenta un sabor amargo, metálico y objetable menor que la muestra comercial baja en sodio y cercanos al $\mathrm{NaCl}$. Estos resultados podrían deberse al menor contenido de sales divalentes en la mezcla $D$, las cuales se han caracterizado por poseer un sabor amargo marcado, seguido de sabor salado, metálico, astringente, agrio y dulce, en orden decreciente de intensidad (Gabriel et al., 2009). En contraste, la mezcla A cuyo contenido de iones de calcio y magnesio es el mayor entre las cuatro mezclas sustitutas, presenta los mayores valores en cuanto a sabor amargo, metálico y sus residuales. 


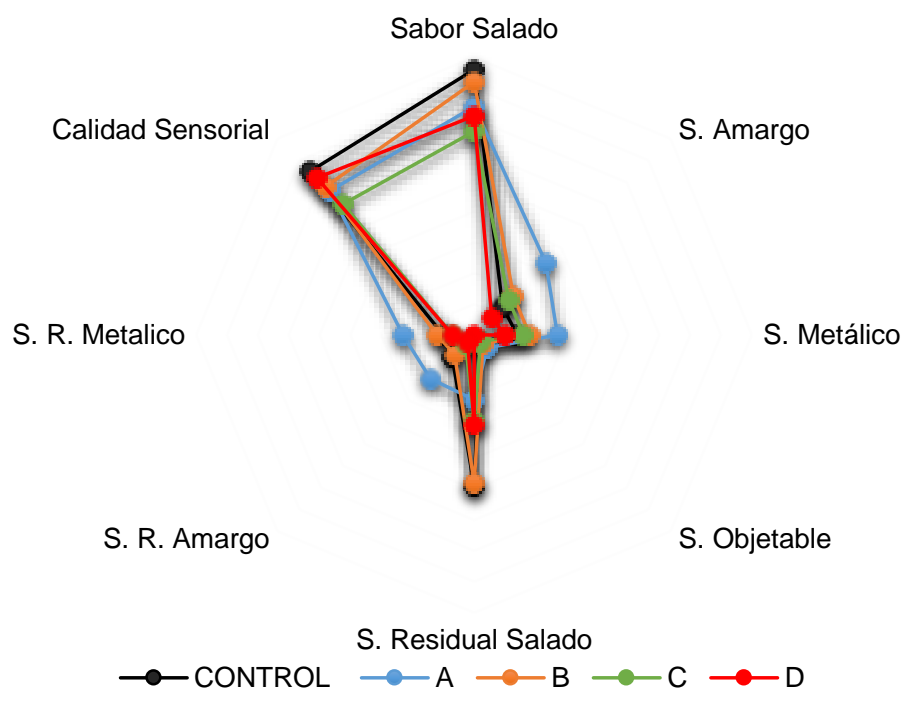

Fig. 1: Perfil sensorial de sabor de las mezclas de sales sustitutas y la sal Vital $40 \%$ menos sodio.

\section{Efecto de la sustitución del $\mathrm{NaCl}$ sobre la calidad microbiológica de los productos}

La Tabla 4 presenta el recuento microbiológico para los productos salchicha y queso elaborados con las mezclas sustitutas y $\mathrm{NaCl}$ como control. Se evidencia que no hay diferencias en el comportamiento del recuento de CF, EC, SA, Mo, S y LM en las diferentes muestras, por otro lado, el recuento de CT, M y L es mayor para el queso elaborado con la mezcla B. Sin embargo, según la NTC 5894 para queso fresco, los recuentos obtenidos para los quesos sustituidos con mezcla de sales y el control se encuentran en el rango permisible, siendo clasificados como quesos de buena calidad microbiológica (ICONTEC, 2011).

Tabla 4: Recuento microbiológico de los productos elaborados con las cuatro mezclas sustitutas y $\mathrm{NaCl}$ como control

\begin{tabular}{|c|c|c|c|c|c|c|c|c|c|c|c|c|}
\hline \multirow[b]{2}{*}{ Mezcla } & \multicolumn{5}{|c|}{ Salchichas } & \multicolumn{7}{|c|}{ Queso } \\
\hline & A & B & C & $D$ & Control & $\begin{array}{l}\text { NTC } \\
1325\end{array}$ & A & B & C & $D$ & Control & $\begin{array}{l}\text { NTC } \\
5894\end{array}$ \\
\hline $\begin{array}{c}\text { CT } \\
\text { (UFC/g) }\end{array}$ & $<10$ & $<10$ & $<10$ & $<10$ & $<10$ & $100-500$ & 140 & 480 & 130 & 50 & 70 & $\begin{array}{l}1000- \\
5000\end{array}$ \\
\hline $\begin{array}{c}\text { CF } \\
(\mathrm{UFC} / \mathrm{g})\end{array}$ & - & - & - & - & - & & $<10$ & $<10$ & $<10$ & $<10$ & $<10$ & \\
\hline $\begin{array}{c}\text { EC } \\
(\mathrm{UFC} / \mathrm{g})\end{array}$ & $<10$ & $<10$ & $<10$ & $<10$ & $<10$ & $<10$ & $<10$ & $<10$ & $<10$ & $<10$ & $<10$ & $<10$ \\
\hline $\begin{array}{c}M \\
\text { (UFC/g) }\end{array}$ & 50 & 190 & 170 & 100 & 180 & $\begin{array}{c}\text { Max } \\
100.000\end{array}$ & 240 & 1300 & 200 & 130 & 130 & \\
\hline $\begin{array}{c}\text { SA } \\
\text { (UFC/g) }\end{array}$ & $<100$ & $<100$ & $<100$ & $<100$ & $<100$ & $<100$ & $\begin{array}{c}<10 \\
0\end{array}$ & $<100$ & $<100$ & $<100$ & $<100$ & $\begin{array}{c}<100- \\
500\end{array}$ \\
\hline $\begin{array}{c}\text { Mo } \\
\text { (UFC/g) }\end{array}$ & - & - & - & - & - & & $<10$ & $<10$ & $<10$ & $<10$ & $<10$ & $10-100$ \\
\hline $\begin{array}{c}\mathrm{L} \\
(\mathrm{UFC} / \mathrm{g})\end{array}$ & - & - & - & - & - & & $<10$ & 10 & 10 & $<10$ & $<10$ & $\begin{array}{l}100- \\
500\end{array}$ \\
\hline $\begin{array}{c}\mathrm{C} \\
(\mathrm{UFC} / \mathrm{g})\end{array}$ & $<10$ & $<10$ & $<10$ & $<10$ & $<10$ & $<10-100$ & - & - & - & - & - & \\
\hline $\mathrm{S} / 25 \mathrm{~g}$ & $\mathrm{~A} / \mathrm{A}$ & $\mathrm{A} / \mathrm{A}$ & $\mathrm{A} / \mathrm{A}$ & $\mathrm{A} / \mathrm{A}$ & $\mathrm{A} / \mathrm{A}$ & $\mathrm{A} / \mathrm{A}$ & $\mathrm{A} / \mathrm{A}$ & $\mathrm{A} / \mathrm{A}$ & $\mathrm{A} / \mathrm{A}$ & $\mathrm{A} / \mathrm{A}$ & $\mathrm{A} / \mathrm{A}$ & $\mathrm{A} / \mathrm{A}$ \\
\hline LM $/ 25 \mathrm{~g}$ & $\mathrm{~A} / \mathrm{A}$ & $\mathrm{A} / \mathrm{A}$ & $\mathrm{A} / \mathrm{A}$ & $\mathrm{A} / \mathrm{A}$ & $\mathrm{A} / \mathrm{A}$ & $\mathrm{A} / \mathrm{A}$ & $\mathrm{A} / \mathrm{A}$ & $\mathrm{A} / \mathrm{A}$ & $\mathrm{A} / \mathrm{A}$ & $\mathrm{A} / \mathrm{A}$ & $\mathrm{A} / \mathrm{A}$ & $\mathrm{A} / \mathrm{A}$ \\
\hline
\end{tabular}

Por otro lado, los análisis microbiológicos realizados a las salchichas elaboradas con las mezclas sustitutas y con el control, no mostraron ninguna diferencia, excepto por el recuento de mesófilos, el cual fue mayor para las mezclas que para el control. Resultados similares han sido encontrados por otros autores, donde no se han reportado diferencias en la calidad microbiológicas de salchichas con sustitución de $\mathrm{NaCl}$ por $\mathrm{KCl}$ (Pacheco et al., 2012).

\section{Efecto de la sustitución del $\mathrm{NaCl}$ sobre las características sensoriales de los productos}

Los atributos sensoriales evaluados por el panel de expertos son mostrados en la Tabla 5 y Fig. 2. Se puede observar que en términos generales las salchichas elaboradas con las mezclas de sales, tienen un perfil sensorial similar al control, destacándose los descriptores de apariencia, sabor y olor característico a 
salchicha, excepto por la elaborada con la mezcla D. Así mismo, los panelistas no lograron detectar olor o sabor objetable en las muestras preparadas con las mezclas A, B y C. Los resultados obtenidos con la mezcla D, podrían deberse a su alto contenido de potasio, dado que se ha demostrado que sustituciones de $\mathrm{NaCl}$ por sales de potasio conllevan a un incremento en el sabor metálico y amargo de los productos (Horita et al., 2014), y en efecto en la Fig. 2 se puede notar que las salchichas elaboradas con esta mezcla, fueron las que presentaron mayor valor en sabor metálico y amargo.

Resultados similares fueron encontrados por Choi et al. (2014), quienes definieron que salchichas frankfurter no muestran diferencias significativas en el sabor y la intensidad de color cuando se sustituye en un $40 \%$ el $\mathrm{NaCl}$ por mezclas que incluyen lactato de $\mathrm{K}$, ascorbato de $\mathrm{Ca}$ y cloruro de $\mathrm{K}$, sin embargo, los panelistas si detectaron diferencias en cuanto la dureza, jugosidad, sabor salado y sabor acido de las salchichas. Por otro lado, algunos autores han definido que no es posible reducir la concentración de $\mathrm{NaCl}$ en salchichas u otros productos cárnicos en más de un $50 \%$ sin que se presenten cambios desfavorables en las características sensoriales del producto (Horita et al., 2011; Pacheco et al., 2012; Horita et al., 2014). En el presente trabajo sin embargo, se logró reducir el porcentaje de $\mathrm{NaCl}$ en cerca de un $63 \%$, con una aceptación de las salchichas por parte de los panelistas de un 100\% para las mezclas A, B y C y de un $83 \%$ para la mezcla $D$.

El análisis sensorial del queso fresco blanco muestra que los elaborados con las mezclas $B$ y $C$ tiene una aceptación del 83,3\%, la cual mayor que la de los quesos elaborados con las mezclas A y D, con 16,7 y $33,4 \%$, respectivamente. Para los panelistas todos los quesos presentan una apariencia y olor característico a queso, excepto por el elaborado con la mezcla A, así mismo, los quesos control, B y C no presentan olor objetable, sabor objetable, sabor amargo o sabor metálico. Pero los quesos con las mezclas $A$ y $D$ presentan percepción del sabor metálico y sabor amargo, esto se puede deber a la presencia de iones de potasio y magnesio.

Tabla 5: Perfil sensorial de los productos elaborados con las cuatro mezclas sustitutas y $\mathrm{NaCl}$ como control.

\begin{tabular}{ccccccccccc}
\hline Descriptor & \multicolumn{9}{c}{ Salchicha } \\
\hline & A & B & C & D & CONTROL & A & B & C & D & CONTROL \\
\hline A & 7,6 & 6,5 & 7,5 & 7,8 & 8,1 & 6,3 & 7,9 & 7,9 & 7,8 & 8,5 \\
OC & 7,8 & 7,4 & 7,5 & 7,5 & 8,1 & 7,0 & 7,7 & 7,1 & 7,9 & 8,0 \\
OO & 0,1 & 0,1 & 0,2 & 0,1 & 0,1 & 0,6 & 0,2 & 0,3 & 0,6 & 0,0 \\
SC & 7,4 & 7,1 & 7,0 & 6,6 & 8,1 & 4,7 & 6,2 & 5,7 & 4,6 & 7,8 \\
SO & 0,1 & 0,1 & 0,3 & 0,6 & 0,0 & 2,1 & 0,6 & 1,0 & 1,5 & 0,0 \\
SS & 2,7 & 2,5 & 2,7 & 2,4 & 2,9 & 1,5 & 1,4 & 1,2 & 1,7 & 2,5 \\
SA & 0,7 & 0,7 & 0,6 & 0,9 & 0,5 & 2,9 & 1,2 & 1,1 & 2,0 & 0,5 \\
SM & 0,7 & 0,6 & 0,8 & 0,9 & 0,5 & 0,6 & 0,3 & 0,5 & 1,3 & 0,1 \\
AC & - & - & - & - & - & 0,7 & 0,4 & 0,6 & 0,8 & 0,4 \\
SG & - & - & - & - & - & 3,2 & 3,2 & 3,6 & 3,3 & 4,1 \\
C & 5,1 & 4,8 & 5,1 & 4,7 & 5,4 & 4,1 & 4,4 & 4,7 & 5,0 & 5,6 \\
Ma & 5,8 & 5,3 & 5,5 & 4,8 & 5,9 & 5,2 & 4,5 & 4,6 & 4,3 & 4,7 \\
CG & 7,2 & 6,9 & 6,8 & 6,3 & 7,8 & 3,5 & 6,1 & 5,7 & 5,3 & 8,0 \\
\% Aceptación & 100 & 100 & 100 & 83,3 & 10 & 16,7 & 83,3 & 83,3 & 33,4 & 100 \\
\hline
\end{tabular}

El queso fresco blanco es un producto de alto consumo en Colombia, pero no en otros países. Por esta razón no es común encontrar estudios sobre dicho producto, aunque sustituciones en el contenido de sodio se han hecho en otros quesos como el mozzarella (Rodrigues et al., 2014) y queso cheddar (Carabante, 2013), donde se reemplazaron hasta el 50\% del contenido de Cloruro de sodio por cloruro de potasio, manteniendo la calidad sensorial aceptable.

Por otro lado, estudios realizados por Rulikowska et al. (2013) revelan que la reducción de la sal en queso impacta de forma negativa eL sabor y textura, resultados similares fueron encontrados por Ganesan et al. (2014) cuándo realizaron reducciones entre el 50 y $60 \%$ de cloruro de sodio en quesos cheddar y mozzarella, donde se evidencio el aumento del sabor amargo, como ocurrió con los quesos elaborados con las mezclas A y D. Mejores resultados fueron obtenidos en la sustitución de hasta un $50 \%$ de $\mathrm{NaCl}$ por $\mathrm{KCl}$ en queso tipo minas, donde los atributos evaluados como la apariencia característica y la calidad general fueron evaluadas por encima de 6, pero el sabor característico, muestra una puntuación alrededor de 5 con una intensidad moderada (Felicio et al., 2016), similar a los resultados encontrados en el actual estudio. En 
el queso fresco Akkawi sustituyeron el $30 \%$ de $\mathrm{NaCl}$ por $\mathrm{KCl}$ obteniendo una calificación alta para el sabor amargo, con una puntuación cercana a 5 (Kamleh et al., 2014), contrario a la encontrada para las mezclas B y $\mathrm{C}$ del presente estudio, que muestran una presencia muy leve del sabor amargo.

a)

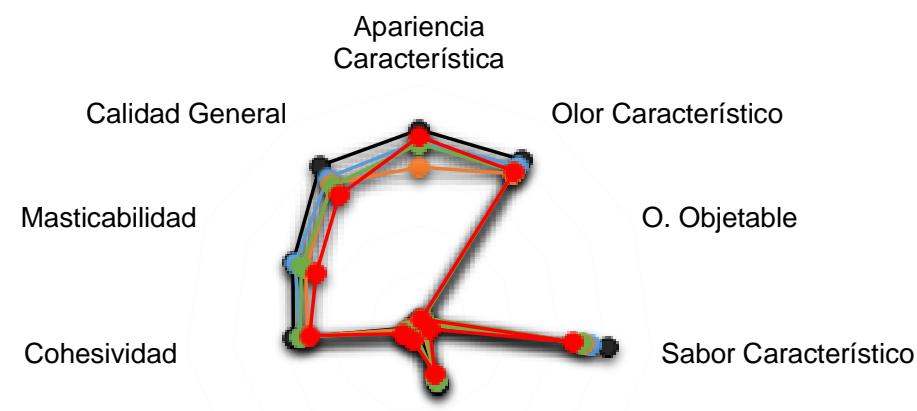

S. Metálico S. Objetable

S. Amargo S. Salado

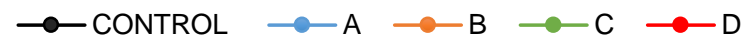

b)

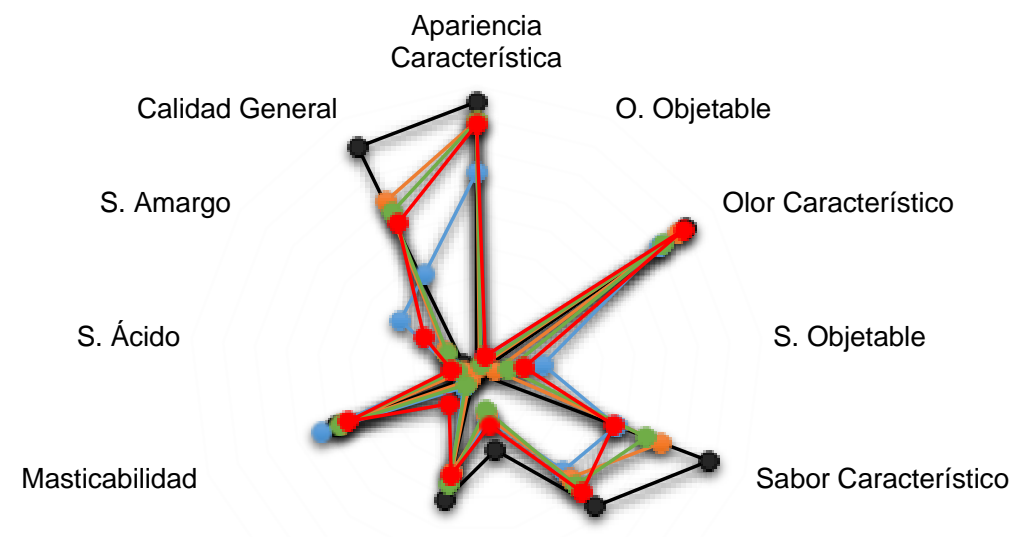

S. Metálico

Cohesividad

S. Graso S. Salado

$\multimap$ CONTROL $\longrightarrow A \multimap B \longrightarrow C \longrightarrow D$

Fig. 2: Perfil sensorial de los productos elaborados con las cuatro mezclas sustitutas y NaCl como control. a) Salchicha premium, b) Queso blanco semiduro-semigraso.

\section{Efecto de la sustitución del $\mathrm{NaCl}$ sobre el color}

En la Tabla 6 se observa que la coordenada cromática $b^{*}$ no presenta diferencias estadísticamente significativas para ninguno de los quesos preparados con las mezclas sustitutas en comparación al control, y que además presenta valores cercanos a otros anteriormente reportados que están entre 19-20 (Carabante, 2013). Las mayores diferencias se dieron en la coordenada $\mathrm{a}^{*}$, donde los quesos elaborados con las mezclas B y D muestran valores ligeramente menores al control, lo cual podría deberse a la mayor proporción de iones de potasio en dichas mezclas, lo cual ha sido reportado por otros autores que han encontrado que la sustitución parcial de $\mathrm{NaCl}$ por $\mathrm{KCl}$ genera disminución en la intensidad de color rojo (Gheisari et al., 2014). El rango presentado para la coordenada $L^{*}$ en los quesos fue de $63,5-68,0$, cuyo valor mayor se obtuvo en el queso elaborado con la mezcla $C$. Estos valores de luminosidad, están en concordancia con valores reportados por otros autores (Carabante, 2013; Gheisari et al., 2014).

A pesar de las diferencias mencionadas para los parámetros de color $a^{*}$ y $L^{*}$, todos los quesos presentaron un color típico que va desde blanco calcáreo hasta el marfil claro, lo cual se pudo corroborar 
dado que los valores de $\Delta \mathrm{E}^{*}$ fueron cercanos a 3 , que se considera una diferencia de color apenas perceptible al ojo humano con respecto al control. Dichos resultados se pueden correlacionar con los resultados sensoriales obtenidos, dado que, según el panel de expertos todos los quesos preparados presentaron una apariencia característica entre moderada y marcada. De igual manera, otros autores han encontrado que sustituciones parciales del $\mathrm{NaCl}$ no generan cambios de color estadísticamente significativos (Gheisari et al., 2014; Kamleh et al., 2014). Otros autores han reportado que una disminución en los niveles de $\mathrm{NaCl}$ en quesos, sin sustitución con otras sales, conllevan a cambios marcados en las características generales del producto. Dicha diferencia se debe a que si no se hace un reemplazo de los iones de sodio en la formulación del queso, se da a una disminución en la fuerza iónica, produciendo cambios en las interacciones entre las proteínas de la leche, tamaño de las micelas de caseína, actividad enzimática y desuerado (Ramirez et al., 2016).

Tabla 6: Parámetros de color $\mathrm{L}^{*} \mathrm{a}^{*} \mathrm{~b}^{*}$ de productos elaborados con las cuatro mezclas sustitutas y $\mathrm{NaCl}$ como control. 'Letras distintas en una misma columna indican diferencia estadística significativa

\begin{tabular}{|c|c|c|c|c|c|}
\hline & Mezcla & $L^{*}$ & $a^{*}(+)$ & $b^{*}(+)$ & $\Delta E^{\star}$ \\
\hline \multirow{5}{*}{ Salchichas } & A & $52,45 \pm 2,18^{a}$ & $16,44 \pm 1,90^{a}$ & $12,28 \pm 1,35^{a}$ & 1,27 \\
\hline & $B$ & $61,94 \pm 0,53^{c}$ & $17,73 \pm 0,53^{b}$ & $13,95 \pm 0,34^{b}$ & 8,74 \\
\hline & C & $57,96 \pm 2,69^{b}$ & $17,27 \pm 1,03^{\mathrm{ab}}$ & $13,22 \pm 0,75^{a}$ & 4,68 \\
\hline & $\mathrm{D}$ & $57,50 \pm 1,16^{b}$ & $16,56 \pm 1,56^{a}$ & $12,70 \pm 0,66^{a}$ & 4,17 \\
\hline & Control & $53,43 \pm 3,13^{a}$ & $17,21 \pm 1,08^{\mathrm{ab}}$ & $12,04 \pm 0,35^{\mathrm{ab}}$ & -- \\
\hline \multirow{5}{*}{ Queso } & $A$ & $63,53 \pm 0,05^{a}$ & $3,44 \pm 0,07^{a b}$ & $21,25 \pm 0,07^{a}$ & 2.69 \\
\hline & B & $66,51 \pm 0,01^{\mathrm{ab}}$ & $3,22 \pm 0,09^{a}$ & $20,58 \pm 0,14^{a}$ & 0.76 \\
\hline & C & $68,05 \pm 0,01^{\mathrm{b}}$ & $3,67 \pm 0,03^{b}$ & $21,56 \pm 0,09^{a}$ & 1.92 \\
\hline & $\mathrm{D}$ & $62,87 \pm 0,10^{\mathrm{a}}$ & $3,23 \pm 0,05^{a}$ & $19,86 \pm 0,06^{a}$ & 3.57 \\
\hline & Control & $66,20 \pm 0,07^{a}$ & $3,71 \pm 0,19^{b}$ & $21,07 \pm 0,17^{a}$ & -- \\
\hline
\end{tabular}

En cuanto a los parámetros de color, las únicas salchichas que no presentan diferencias significativas con el control son las elaboradas con la mezcla $\mathrm{A}$, mientras que las otras mezclas presentan diferencias en alguna de las coordenadas de color, lo cual está en concordancia con el análisis sensorial realizado, donde los panelistas atribuyen a todas las salchichas una apariencia característica marcada. Se pueden observar también, que los valores para la coordenada $\mathrm{a}^{*}$ están entre 16 y 18, lo cual corresponde al color rojo típico de este tipo de productos (Horita et al., 2014; Marchetti et al., 2015).

Las salchichas elaboradas con las mezclas B, C y D, presentan mayor luminosidad que el control, mientras que las de la mezcla A no la presentan. Hallazgos similares han sido presentados por Horita et al. 2014, quienes encontraron que la reducción del sodio en salchichas frankfurter conlleva a un aumento en la luminosidad de las mismas. Algunos autores como Boyle et al. (1994), estudiaron salchichas fortificadas con calcio, concluyendo que la luminosidad aumentó cuando el calcio fue incluido en la formulación de las salchichas, sin embargo en el presente estudio la mezcla $A$ que no presentó diferencias en la luminosidad de las salchichas con respecto al control, es precisamente la mezcla que mayor contenido de $\mathrm{Ca}^{2+}$ presenta. Estos resultados sugieren que la luminosidad de las salchichas se puede ver afectada no solo por el contenido de calcio en la mezcla sustituta, sino por los demás iones presentes. Lo cual fue reportado también por Gimeno et al. (2001), quien concluyó que la naturaleza química de los componentes empleados como sustitutos del $\mathrm{NaCl}$ tiene influencia en el desarrollo del color aun cuando ellos aporten una fuerza iónica equivalente.

\section{Efecto de la sustitución del $\mathrm{NaCl}$ sobre la textura instrumental}

Para evaluar el efecto de la sustitución de $\mathrm{NaCl}$ sobre la textura del queso fresco y la salchicha tipo premium se realizó un análisis de perfil de textura (TPA). La Tabla 7 muestra que el parámetro elasticidad para el control y los quesos elaborados con las mezclas no presentan diferencias, por otro lado, la dureza, la gomosidad y la masticabilidad muestra diferencia con respecto al control, así como la cohesividad es diferente solo para el queso elaborado con la mezcla C. Resultados similares para queso fresco fueron obtenidos por Guzmán et al. (2015) para la dureza, cohesividad, gomosidad, y elasticidad. Pero la masticabilidad fue más alta, con resultados entre 30 y $60 \mathrm{~N}$. Respecto a la dureza, se observa que los quesos sustituidos con mezclas de sales disminuye, este mismo comportamiento fue encontrado por Murtaza et al. (2014) cuando se reduce la sal en la elaboración de queso cheedar. Diferentes autores han estudiado el efecto de la sustitución de sodio por otros iones en la elaboración de quesos y han determinado 
el alto efecto que tiene dicho reemplazo sobre la textura del producto, principalmente por la presencia de iones de calcio, los cuales vinculados en exceso a la molécula de paracaseína, causan una incorporación excesiva de iones de sodio produciendo un queso más suave (Ramirez et al., 2016).

En productos cárnicos emulsificados como las salchichas, la textura es uno de los atributos sensoriales más importantes y está directamente relacionado con la capacidad de retención de agua de la matriz cárnica, la cual está influenciada por la fuerza iónica y las propiedades funcionales de las proteínas (Hamm, 1986). Así la sal juega un rol importante en la extracción de proteínas miofibrilares dado que incrementa la hidratación y la capacidad vinculante de agua por la fuerte unión de los iones clorhídricos a las proteínas. De esta manera, cuando el $\mathrm{NaCl}$ es reducido, la cantidad de proteínas miofibrilares solubles pueden también disminuir, conllevando a una menor capacidad de retención de agua y fuerza del gel (Desmond, 2006).

Tabla 7: Parámetros de textura de los productos elaborados con las cuatro mezclas sustitutas y $\mathrm{NaCl}$ como control

\begin{tabular}{ccccccc}
\hline & Mezcla & Dureza $(N)$ & Cohesividad & Gomosidad $(N)$ & Masticabilidad $(N)$ & Elasticidad \\
\hline & A & $20,22 \pm 1,25^{\mathrm{a}}$ & $0,77 \pm 0,02^{\mathrm{b}}$ & $15,54 \pm 0,80^{\mathrm{a}}$ & $13,48 \pm 0,05^{\mathrm{a}}$ & $0,87 \pm 0,02^{\mathrm{ab}}$ \\
Salchichas & B & $24,89 \pm 1,35^{\mathrm{c}}$ & $0,76 \pm 0,01^{\mathrm{a}}$ & $18,81 \pm 0,99^{\mathrm{c}}$ & $16,30 \pm 0,06^{\mathrm{c}}$ & $0,87 \pm 0,01^{\mathrm{ab}}$ \\
& C & $24,91 \pm 0,98^{\mathrm{c}}$ & $0,76 \pm 0,01^{\mathrm{a}}$ & $18,82 \pm 0,71^{\mathrm{c}}$ & $16,54 \pm 0,04^{\mathrm{c}}$ & $0,88 \pm 0,01^{\mathrm{bc}}$ \\
& D & $22,46 \pm 3,39^{\mathrm{b}}$ & $0,77 \pm 0,01^{\mathrm{b}}$ & $17,39 \pm 2,55^{\mathrm{b}}$ & $15,07 \pm 0,16^{\mathrm{b}}$ & $0,87 \pm 0,02^{\mathrm{a}}$ \\
Control & $22,08 \pm 0,85^{\mathrm{b}}$ & $0,78 \pm 0,02^{\mathrm{b}}$ & $17,12 \pm 0,46^{\mathrm{b}}$ & $15,19 \pm 0,03^{\mathrm{b}}$ & $0,89 \pm 0,01^{\mathrm{c}}$ \\
\hline A & $22,04 \pm 3,07^{\mathrm{a}}$ & $0,71 \pm 0,02^{\mathrm{a}}$ & $15,70 \pm 2,29^{\mathrm{a}}$ & $11,51 \pm 1,41^{\mathrm{a}}$ & $0,84 \pm 0,09^{\mathrm{a}}$ \\
& B & $19,21 \pm 1,46^{\mathrm{a}}$ & $0,69 \pm 0,04^{\mathrm{a}}$ & $13,25 \pm 1,45^{\mathrm{b}}$ & $11,02 \pm 0,40^{\mathrm{a}}$ & $0,83 \pm 0,02^{\mathrm{a}}$ \\
& C & $21,85 \pm 3,00^{\mathrm{a}}$ & $0,66 \pm 0,03^{\mathrm{b}}$ & $14,40 \pm 1,63^{\mathrm{a}, \mathrm{b}}$ & $11,06 \pm 0,98^{\mathrm{a}}$ & $0,83 \pm 0,05^{\mathrm{a}}$ \\
& D & $22,60 \pm 1,93^{\mathrm{a}}$ & $0,72 \pm 0,03^{\mathrm{a}}$ & $16,28 \pm 1,60^{\mathrm{a}}$ & $12,36 \pm 1,04^{\mathrm{a}}$ & $0,82 \pm 0,03^{\mathrm{a}}$ \\
& Control & $27,75 \pm 5,05^{\mathrm{b}}$ & $0,70 \pm 0,02^{\mathrm{a}}$ & $19,33 \pm 3,29^{\mathrm{c}}$ & $15,77 \pm 0,79^{\mathrm{b}}$ & $0,82 \pm 0,01^{\mathrm{a}}$ \\
\hline
\end{tabular}

El perfil de textura de las salchichas se ve afectado con la sustitución del $\mathrm{NaCl}$ por las mezclas, excepto por las salchichas elaboradas con la mezcla D. Resultados similares fueron reportados por Marchetti et al. (2015), quienes encontraron que la dureza, elasticidad, adhesividad y masticabilidad de salchichas bajas en grasa, fue significativamente afectada con el remplazo parcial de $\mathrm{NaCl}$ por $\mathrm{KCl}$ y sodio tripolifosfato.

Las salchichas elaboradas con la mezcla $\mathrm{D}$, la cual contiene mayor proporción de $\mathrm{K}^{+}$, no presentaron diferencias estadísticamente significativas en cuanto a la dureza, cohesividad, gomosidad y masticabilidad con respecto al control, a diferencia de las elaboradas con las otras mezclas sustitutas que contienen mayor proporción de $\mathrm{Ca}^{2+}$ y $\mathrm{Mg}^{2+}$. Lo anterior podría deberse a que la fuerza iónica y las propiedades químicas de las sales de potasio, son similares a las del $\mathrm{NaCl}$, mientras que muchos estudios han reportado que sales divalentes pueden reducir el nivel de las proteínas funcionales, resultando en emulsiones inestables (Horita et al., 2014).

Las salchichas formuladas con las mezclas B y C muestran un aumento en la dureza, cohesividad, gomosidad y masticabilidad con respecto al control, mientras que la formulación con la mezcla $A$ conlleva a una disminución en los mismos. Horita et al. (2014) encontraron también un aumento en la dureza de salchichas frankfurters cuando el $\mathrm{NaCl}$ es sustituido en un 25 y $50 \%$ por $\mathrm{CaCl}_{2}$ o $\mathrm{KCl}$. Así mismo Mora et al. (2014) encontraron un aumento en la dureza, masticabilidad y cohesividad de salchichas fermentadas reducidas en sodio. De acuerdo a estos autores, el incremento en la dureza es atribuido a la reducción de los enlaces de agua durante la cocción. Sin embargo, otros autores han reportado disminución en el valor de dureza, cohesividad, masticabilidad y gomosidad en productos cárnicos emulsificados con la sustitución del $\mathrm{NaCl}$. Así Horita et al. (2011) encontraron que la sustitución de un $50 \%$ de $\mathrm{NaCl}$ por $\mathrm{KCl}$, conlleva a menores valores en la dureza y cohesividad de mortadela. Choi et al. (2014) mostraron una disminución en la gomosidad de salchichas frankfurter formuladas con una sustitución de un 10\% de lactato de potasio y $30 \%$ ascorbato de calcio en remplazo del $\mathrm{NaCl}$. Pacheco et al. (2012) reportaron que una reducción en un $75 \%$ de $\mathrm{NaCl}$ causa reducción en la dureza, gomosidad y masticabilidad de salchichas. De esta manera, se puede ver que en la literatura se muestran diferentes resultados para los perfiles de textura de salchichas reducidas en sodio dependiendo del tipo de carne, los niveles de reducción de $\mathrm{NaCl}$ y el componente usado para su sustitución. 


\section{CONCLUSIONES}

El contenido en iones de las sales en estudio afecta de manera significativa el perfil de sabor de las soluciones salinas y el perfil de textura y de sabor de los productos elaborados, pero no afecta la calidad microbiológica ni el color instrumental de los productos. Este efecto además depende del tipo de producto en que sean utilizadas las sales bajas en sodio. En general es posible la sustitución del $63 \%$ de $\mathrm{NaCl}$ por las mezclas A y B para la elaboración de salchichas y quesos frescos, respectivamente, sin afectar sus características físicas, microbiológicas y sensoriales.

\section{AGRADECIMIENTOS}

Los autores agradecen a la Empresa Brinsa S.A.S y al Programa de Gestión Tecnológica de la Universidad de Antioquia, por el apoyo financiero entregado para la ejecución del proyecto, así mismo a Ingeniero Juan Camilo Arango Bivoloti y al personal de Brinsa S.A.S que participaron en diferentes actividades del mismo.

\section{REFERENCIAS}

Boyle, E.A.E.; P.B. Addis y R.J. Epley, Calcium Fortified, Reduced Fat Beef Emulsion Product, Journal of Food Science, 59(5), 928-932 (1994)

Carabante, K.M., Effect of Bitterness Blockers and Salt Substitutes on the Quality of Low Sodium White Cheddar Cheese, Tesis de Magister, Louisiana State University, Luisiana, Estados Unidos (2013)

Choi, Y.M.; K.C. Jung; H.M. Jo; K.W. Nam; J.H. Choe; M.S. Rhee y B.C. Kim, Combined effects of potassium lactate and calcium ascorbate as sodium chloride substitutes on the physicochemical and sensory characteristics of low-sodium frankfurter sausage, Meat Science, 96(1), 21-25 (2014)

Desmond, E., Reducing salt: A challenge for the meat industry, Meat Science, 74(1), 188-196 (2006)

Felicio, T.L.; E.A. Esmerino; A.G. Cruz; L.C. Nogueira; R.S.L. Raices, R. Deliza y M.A.R. Pollonio, Cheese. What is Its Contribution to the Sodium Intake of Brazilians? Appetite, 66, 84-88 (2013)

Felicio, T.L.; E.A. Esmerino; V.A.S. Vidal; L.P. Cappato; R.K.A. Garcia; R.N. Cavalcanti y A.G. Cruz, Physico-Chemical Changes During Storage and Sensory Acceptance of Low Sodium Probiotic Minas Cheese Added with Arginine, Food Chemistry, 196, 628-637 (2016)

Fitzgerald, E. y J. Buckley, Effect of Total and Partial Substitution of Sodium Chloride on the Quality of Cheddar Cheese, Journal of Dairy Science, 68(12), 3127-3134 (1985)

Gabriel, A.S.; H. Uneyama; T. Maekawa y K. Torii, The calcium-sensing receptor in taste tissue, Biochemical and Biophysical Research Communications, 378(3), 414-418 (2009)

Ganesan, B.; K. Brown; D.A. Irish; C. Brothersen y D.J. McMahon, Manufacture and Sensory Analysis of Reduced and low Sodium Cheddar and Mozzarella Cheeses, J. of Dairy Science, 97(4), 1970-1982 (2014)

Gheisari, H.R.; M. Aminlari; N. Sabbagh y M. Moraveji, Chemical and microbiological changes during ripening of Iranian salt-substituted probiotic white cheese, Carpathian Journal of Food Science and Technology, 6(2), 102-115 (2014)

Gimeno, O.; I. Astiasarán y J. Bello, Calcium Ascorbate as a Potential Partial Substitute for $\mathrm{NaCl}$ in Dry Fermented Sausages: Effect on Colour, Texture and Hygienic Quality at Different Concentrations, Meat Science, 57(1), 23-29 (2001)

Guzmán, L. E.; C. Tejada; Y.J. De la Ossa y C.A. Rivera, Análisis Comparativo de Perfiles de Textura de Quesos Frescos de Leche de Cabra y Vaca, Biotecnología en El Sector Agropecuario y Agroindustrial, 13(1), 139-148 (2015)

Hamm, R., Muscle as food. In P. J. Bechtel (Ed.), 135-199. Academic Press, Orlando (1986)

Horita, C.N.; V.C. Messias; M.A. Morgano; F.M. Hayakawa y M.A.R. Pollonio, Textural, microstructural and sensory properties of reduced sodium frankfurter sausages containing mechanically deboned poultry meat and blends of chloride salts, Food Research International, 66, 29-35 (2014) 
Horita, C.N.; M.A. Morgano; R.M.S. Celeghini y M.A.R. Pollonio, Physico-chemical and sensory properties of reduced-fat mortadella prepared with blends of calcium, magnesium and potassium chloride as partial substitutes for sodium chloride, Meat Science, 89(4), 426-433 (2011)

ICONTEC, NTC 5894, Productos lácteos. Queso Fresco, 1-18, Bogotá, Colombia (2011)

ICONTEC, NTC 1325, Industrias Alimentarias. Productos Cárnicos Procesados No Enlatados, 1-32, Bogotá, Colombia (2008)

ICONTEC, NTC 3932, Análisis Sensorial. Identificación y Selección de Descriptores para Establecer un Perfil Sensorial por una Aproximación Multidimensional, 1-31, Bogotá, Colombia (1996)

ICONTEC, NTC 5328, Análisis Sensorial. Directrices para el Uso de Escalas de Respuesta Cuantitativas, 113, Bogotá, Colombia (2004)

Ifat, C. y B. Ofir, Reduction in Sodium Content of Fresh, Semihard Tzfat Cheese Using Salt Replacer Mixtures: Taste, Texture and Shelf Life Evaluation, International J. of Dairy Technology, 69, 1-11 (2016)

Kamleh, R.; A. Olabi; I. Toufeili; H. Daroub; T. Younis y R. Ajib, The Effect of Partial Substitution of NaCl With $\mathrm{KCl}$ on The Physicochemical, Microbiological and Sensory Properties of Akkawi Cheese, Journal of the Science of Food and Agriculture, 95(9), 1940-1948 (2014)

Marchetti, L.; N. Argel; S.C. Andrés y A.N. Califano, Sodium-reduced lean sausages with fish oil optimized by a mixture design approach, Meat Science, 104, 67-77 (2015)

Mora, H.; X. Serra; M.D. Guàrdia y J. Arnau, Effect of reducing and replacing pork fat on the physicochemical, instrumental and sensory characteristics throughout storage time of small caliber non-acid fermented sausages with reduced sodium content, Meat Science, 97(1), 62-68 (2014)

Murtaza, M.A.; N. Huma; A. Sameen; M.S. Murtaza; S. Mahmood; G. Mueen-ud-Din y A. Meraj, Texture, flavor, and sensory quality of buffalo milk Cheddar cheese as influenced by reducing sodium salt content, Journal of Dairy Science, 97(11), 6700-6707 (2014)

Pacheco, W.A.; C.E. Arias y D.A. Restrepo, Efecto de la Reducción de Cloruro de Sodio sobre las Características de Calidad de una Salchicha Tipo Seleccionada, Revista Facultad Nacional de Agronomía, 65(12), 6779-6787 (2012)

Ramirez, J.S.; J. Aguirre; V.A. Aristizabal y S. Castro, La sal en el queso: diversas interacciones, Agronomía Mesoamericana, 28(1), 303-316 (2016)

Rodrigues, J.F.; C.S. Gonçalves; R.C. Pereira; J.D.S. Carneiro y A.C.M. Pinheiro, Utilization of temporal dominance of sensations and time intensity methodology for development of low-sodium Mozzarella cheese using a mixture of salts, Journal of Dairy Science, 97(8), 4733-44 (2014)

Rulikowska, A.; K.N. Kilcawley; I.A. Doolan; M. Alonso-Gomez; A.B. Nongonierma; J.A. Hannon y M.G. Wilkinson, The impact of reduced sodium chloride content on Cheddar cheese quality, International Dairy Journal, 28(2), 45-55 (2013)

Soares, C.; A.L. Fernando y B. Mendes, The effect of lowering salt on the physicochemical, microbiological and sensory properties of São João cheese of Pico Island, International Journal of Dairy Technology, 68(3), 409-419 (2015)

Zhou, B.; J. Webster; L.Y. Fu; H.L. Wang; X. M. Wu; W.L. Wang y J.P. Shi, Intake of low sodium salt substitute for 3 years attenuates the increase in blood pressure in a rural population of North China-A randomized controlled trial, International Journal of Cardiology, 215, 377-382 (2016) 
